\title{
Enhanced Unsupervised Noise Cancellation using Angular Resampling for Planetary Bearing Fault Diagnosis
}

\author{
F. Bonnardot \\ LASPI, IUT de Roanne, 20, avenue de Paris, 42334 Roanne Cedex, France
}

\author{
R. B. Randall ${ }^{\dagger}$ \\ School of Mechanical and Manufacturing Engineering, UNSW, Sydney NSW 2052, Australia
}

\author{
J. Antoni \\ University of Technologie of Compiègne, 60205 Compiègne, France
}

(Received 20 August 2003; accepted 11 March 2004)

\begin{abstract}
In this paper, some techniques for bearing diagnosis are reviewed (unsupervised angular resampling and noise cancellation, envelope analysis) and applied in combination for the first time to solve a particularly difficult diagnostic problem. Unsupervised noise cancellation exploits the periodicity of gear signals. Since the vibrations from gears are periodic in the angular domain, we propose an enhanced method that uses an unsupervised ordertracking algorithm to perform noise cancellation in the angular domain rather than in the time domain. This method is then applied to bearing fault diagnosis of a planetary bearing in a helicopter gearbox. Due to random speed fluctuation, unsupervised noise cancellation initially did not separate the gear and bearing signals. However, the enhanced noise cancellation, which includes a pre-treatment to suppress speed fluctuation based on phase demodulation of gearmesh frequencies, without the need for a tachometer signal, provides better results. Finally the denoised signal was studied using the envelope analysis technique, and the bearing fault frequency was then detected. Without proper noise cancellation this was not readily detectable in the spectrum noise.
\end{abstract}

${ }^{\dagger}$ Member of the International Institute of Acoustics and Vibration (IIAV)

\section{INTRODUCTION}

The bearings of planetary gears provide one of the most difficult scenarios for detection and diagnostics of bearing faults, since the fault signals must pass through a tortuous and time-varying path to arrive at external measurement points where they can be detected. In the case of helicopter gearboxes, this is made even more difficult by the fact that strong background masking signals exist over the full acoustic frequency range, in particular from gears which convert an input shaft frequency at gas turbine speed of typically 350 $\mathrm{Hz}$ to a rotor output speed of typically $5 \mathrm{~Hz}{ }^{1}$

The first step in analysing bearing signals is to remove the contributions from the gears. Classically, in normal gearboxes, this is done by using a simple band pass filter that exploits their different frequency ranges. ${ }^{2}$ However, with helicopter gearbox signals, it is usually necessary to first remove the masking signals from gears before the bearing signals can be analysed. In a previous article by $\mathrm{Ho}^{3}$ the self-adaptive noise cancellation technique was used to improve envelope analysis results, but this requires the gear signals to be deterministic and phase-locked to shaft speeds. If the shaft speeds vary somewhat, it may be necessary to resample the signals on an angular rather than temporal basis (so-called order tracking), to force the gear signals to be deterministic. This normally requires the use of a shaft phase-locked tachometer signal to perform the angular resampling.
The aim of this article is to show how the angular resampling can be performed from the signal itself without the requirement of a tachometer signal, thus combining unsupervised noise cancellation with angular resampling (i.e., effectively eliminating the speed fluctuation) to enhance the quality of the separation. A newly developed separation technique is utilised, which is much more efficient than that used by $\mathrm{Ho}^{3}$

After presentation of the separation technique, its application to a particularly difficult diagnostic problem is then detailed.

\section{BEARING SIGNAL SEPARATION METHODS (1 SENSOR)}

Separation methods exploit the different nature of bearing and gear signals. First, we will review some analysis tools (envelope analysis, unsupervised noise cancellation). Next, we will propose a new enhanced method which combines angular resampling with unsupervised noise cancellation and envelope analysis.

\subsection{Envelope Analysis}

McFadden and $\mathrm{Smith}^{4}$ made a model for a bearing fault (single point defect) (see Fig. 1):

- The contact between the defective surface and the other moving surface is modelled by an impulse source $d(t)$ (whose repetition frequency depends on the type of fault), 